\title{
Threat Description for the PP by Using the Concept of the Assets Protected by TOE
}

\author{
Tai-hoon Kim ${ }^{1}$, Byung-gyu No ${ }^{1}$, and Dong Chun Lee ${ }^{2}$ \\ 1 KISA, 78, Garak-Dong, Songpa-Gu, Seoul, Korea \\ \{taihoon, nono\}akisa.or.kr \\ 2 Dept. of Computer Science Howon Univ., Korea \\ ldchesunny.howon.ac.kr
}

\begin{abstract}
Evaluation has been the traditional means of providing assurance and is the basis for prior evaluation criteria documents such as ITSEC. The Common Criteria (CC) defines a Protection Profile (PP) that defines the security environments and specifies the security requirements and protections of the product to be evaluated. The security environments consist of assumptions, threats, and organizational security policies, so the editor of the PP must describe the threats for the PP. In this paper, we propose a method for the description of the threats for the PP by introducing the concept of the assets protected by Target of Evaluations (TOE).
\end{abstract}

\section{Introduction}

The CC philosophy is to provide assurance based upon an evaluation of the IT product or system that is to be trusted. Evaluation has been the traditional means of providing assurance.

There are many evaluation criteria. The Trusted Computer System Evaluation Criteria (TCSEC), the European Information Technology Security Evaluation Criteria (ITSEC), and the Canadian Trusted Computer Product Evaluation Criteria (CTCPEC) existed, and they have evolved into a single evaluation entity, the CC. The CC for Information Technology Security Evaluation is the result of an international project involving NIST and the National Security Agency (NSA), on behalf of the United States and security organizations in Canada, France, Germany, the Netherlands, and the United Kingdom. These countries have worked in close cooperation with the International Organization for Standard (IOS) 15408 [1][2][3][4].

The CC is a standard for specifying and evaluating the security features of IT products and systems, and is intended to replace previous security criteria such as the TCSEC. This evaluation process establishes a level of confidence that the security functions of such products and systems, and the assurance measures applied to them, must meet.

The CC defines a PP that specifies the security requirements and protections of the product to be evaluated. If someone wants to select IT systems which 
meet his requirements for security, he can use the PP to express his security requirements.

Those who want to express their security requirements using the $\mathrm{PP}$ must first define the security environments which consist of assumptions, threats, and organizational security policies, and connect the security environments to the security objectives and security requirements in the $\mathrm{CC}$. When the writing the threat phrases for the PP, we use the production rule of multi-stage graph. But the assets and the attack methods increase rapidly, the count of the total threat phrases increases too and some new method for reducing these is needed.

In this paper we introduce the concept of the 'assets protected by TOE' and propose a new method for reducing the count of the threat phrases by using that concept.

\section{Threat Description for the PP}

\subsection{Protection Profile}

A PP defines an implementation-independent set of IT security requirements for a category of TOEs. Such TOEs are intended to meet common consumer needs for IT security. Consumers can therefore construct or cite a PP to express their IT security needs without reference to any specific TOE.

The purpose of a PP is to state a security problem rigorously for a given collection of systems or products (known as the TOE) and to specify security requirements to address that problem without dictating how these requirements will be implemented. For this reason, a PP is said to provide an implementationindependent security description. A PP thus includes several related kinds of security information (See the Fig. 1).

A description of the TOE security environment which refines the statement of need with respect to the intended environment of use, producing the threats to be countered and the organizational security policies to be met in light of specific assumptions.

\subsection{Threating Be Identified}

A 'threat' is simply an undesirable event, which is characterized in terms of a threat agent, a presumed attack method, an identification of the asset under attack, and so on. In order to identify what the threats are, we therefore need to answer the following questions:

- What are the assets that require protection? : The assets subject to the attack (e.g., sensitive data),

- Who or what are the threat agents? : The threat agent (e.g., an authorized user of the TOE),

- What attack methods or undesirable events do the assets need to be protected from? : The attack methods employed (e.g., impersonation of an authorized user of the TOE). 


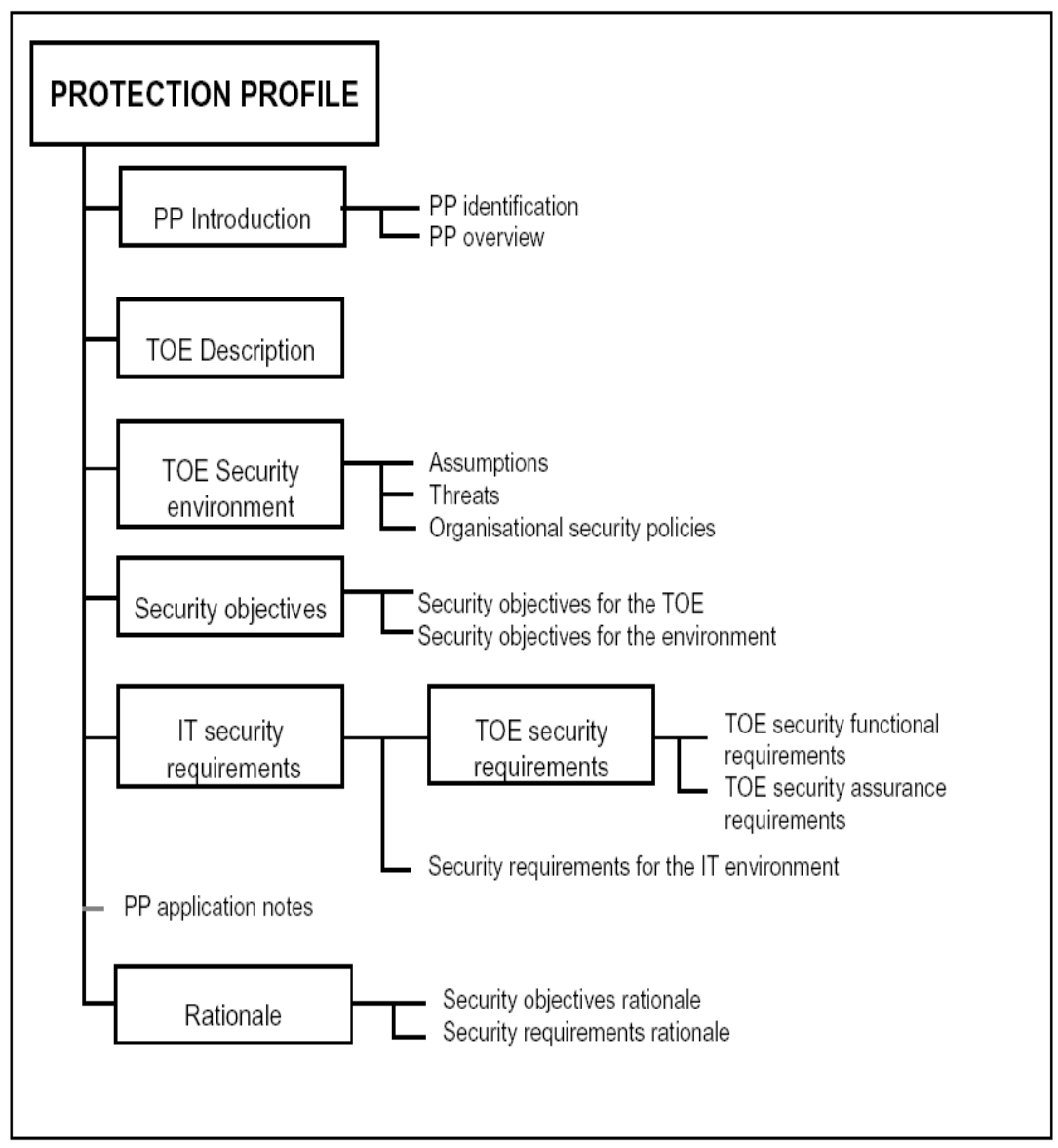

Fig. 1. Protection Profile content

\subsection{Threats of the Wireless Networks}

There are many kinds of 'threat' in the wireless networks and these are expressed in many books [7][8][9] or web-sites. But these threats are not the unusual things which are different from the threats other systems have. In fact, the security functional requirements in the Part 2 of $\mathrm{CC}$ are not restricted to the network systems as though they are the major target for development of the PP.

For example, there is the FPT_TDC family in the Part 2 of the CC which means 'Inter-TSF TSF data consistency'. In some cases, especially in a distributed or composite system environment, a TOE may need to exchange TSF data with another trusted IT product. The FPT_TDC family defines the requirements for sharing and consistent interpretation of these attributes between the TSF of the TOE and a different trusted IT product. But this family does not comment whether the environment is wire or wireless networks. In fact, the 
difference between wire and wireless networks is not important for meeting of the requirements of the FTP_TDC.

The security functional requirements of the $\mathrm{CC}$ are described as high level form independent on a special technical factor. Therefore, they can be applied to many kinds of systems and security environments.

\section{Describing the Threats in the PP}

In the PP, in order to ensure we have a 'concise' statement of threats, the threat descriptions should be 'disjoint' as far as possible. In other words, there should be minimal overlap between different threats. This will help avoid potential confusion on the part of the reader of the PP as well as helping to simplify the $\mathrm{PP}$ rationale by avoiding needless repetition.

If we specify all threats at the same level of detail, the overlaps between threats can be more easily avoided. For example, it's not good that a threat description contains a detailed attack method against a specific asset if this is a specific attack scenario that is already associated with a more general threat stated elsewhere in the PP. Therefore, if we want to describe the threats for the $\mathrm{PP}$, more considerations are needed.

Actually, all IT systems and communications channels face security threats that can compromise the systems themselves, the services they provide, the data stored on, and the data transmitted between them. For describing the threats for the PP, next items must be identified.

\subsection{Identifying the Assets}

In the case of writing threat phrases, it is very important to categorize the asset. When considering the assets of systems, we first think the factors threat agents want to compromise. Maybe we can use the next items as assets of the wireless networks.

- Wireless devices or systems

- Service that wireless networks provide

- Data stored on the wireless networks

- Data transmitted between the wireless networks.

- Other network resources.

In fact, these are very simple categorization of asset. For example, let's consider a wireless phone. The device user has is an important asset and must be protected from unauthorized user. If the device provides a commerce service, the service is protected from misuse, abuse, and so on. And the phone numbers or keys or passwords stored in the device are critical things and if these data transmitted to other system, they must be checked for confidentiality and integrity.

As mentioned earlier, the categorization of asset is very important thing to write threat phrases for the PP, because the threat may be described by using the assets. 


\subsection{Identifying the Threat Agents}

Threat agents may either be human or non-human, and we can use the next items as threat agents of the wireless networks.

- Authorized users or owners of the wireless networks.

- Unauthorized users of the wireless networks.

- Attackers or intruders or hackers.

- IT entities.

When we consider the threats described in the PP, authorized users or owners of the wireless device is looked on as threat agent because they can commit abuse or unauthorized use.

In the field of requirement engineering, the concept of user is inclined to be restricted to human. But in the CC, the concept of user is extended to the IT entities and this may be correct thing because the malicious codes or viruses can be the user of the function.

\subsection{Identifying the Attack Methods}

Having identified the assets to be protected and the threat agents which may be the subject of attack, the next step is to identify the possible attack methods which could lead to a compromise of the assets.

This will be based on what is known regarding the TOE security environment. There is a very important thing must be considered. If we want to consider all attack methods already known, maybe it is impossible because the attack methods are too various and created or found everyday. Therefore, if we want to describe the threats about the TCP/IP, should use the phrase 'by using TCP/IP vulnerabilities already known' not dividing as like flooding, spoofing, DoS, and so on.

\section{Description of Threat in the PP}

After identifying the assets, threat agents, and attack method, we can describe the threats for the PP. For example, we can describe the threats concerned with the confidentiality of assets in the PP as like this:

- Threat agents may discover wireless networks by unauthorized methods,

- Threat agents may disclose the services provided by unauthorized methods,

- Threat agents may disclose the data stored by unauthorized methods,

- Threat agents may disclose the data transmitted by unauthorized methods,

- Threat agents may disclose the systems or network resources by unauthorized methods,

- Etc.

But if we describe the threats as like these, there can be too many threat phrases in the PP, so a new method defining the threat is needed. We did not consider various threat agents and the threat methods above. If we divide the threat agents as authorized user and unauthorized user, threats may be two times as above. 


\subsection{Identifying of the Threats Considered the Composition of the Assets, the Threat Agents, and the Attack Methods}

Many cases, when we describe the threats in the PP, we can use the 'production rule of multi-stage graph' (See the Fig. 2). In other words, the threats can be described by the combination of the assets, the threat agents, the attack methods, and so on. (But the Fig. 2 is not complete form.) Therefore, there can be very many phrases for the threats, and these phrases are the headache of the editors of PP.

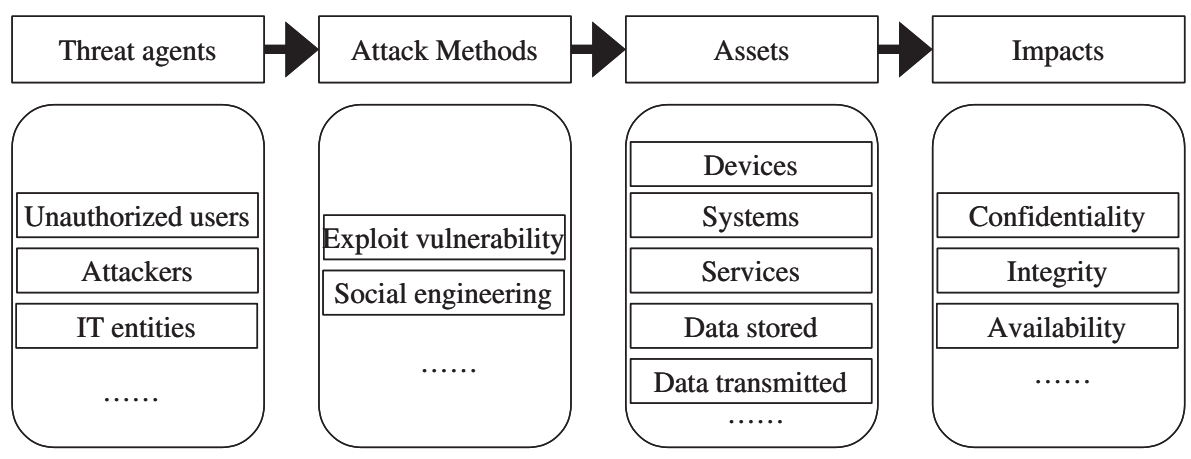

Fig. 2. Threat production rule of multi-stage graph (Example)

As you saw in the Fig. 2, the count of the threat phrase is decided by the next numerical formula:

Count of threat $=$ Threat agent $*$ Attack method $*$ asset $*$ Impact $*$ Etc.

Therefore, we must re-consider and minimize the terms of numerical formula mentioned above for the efficient writing of threat phrases. In many cases, we can solve this problem by adjusting the 'Assumption' and 'Organizational Security Policy (OSP)', but this method is 'minimizing the threat agent' or 'reducing the attack methods possible'. When developing the systems which consider the security, most functional requirements are implemented by the threats not assumption or OSP. If we describe the security environments with many assumptions and OSPs, many of the functional requirements can't be implemented correctly and these systems may not cover the real threats.

\subsection{Assets Protected by TOE}

We propose a method for minimizing the assets of IT systems including wireless systems to reduce the count of threat phrases. As mentioned earlier, maybe we can use the next items as assets of the wireless networks. 
- Wireless devices or systems,

- Service that wireless systems provide,

- Data stored on the wireless systems,

- Data transmitted between the wireless systems,

- Other network resources

In the aspects of the security requirements and the evaluation of the IT systems, we can re-identify the assets as like;

- TOE (target of evaluation),

- Assets protected by TOE

- Systems not included in the TOE

- Services provided

- Data stored

- Data transmitted

- etc.

This method for identifying of assets is available for the PP, because the identifying of the security environments is connected to the security functional requirements included in the $\mathrm{CC}$.

For example, let's describe the threat concerned with the confidentiality of assets (In this example, we didn't classify the threat agents and the attack methods, and applied 'the assets protected by TOE' to the description).

- Threat agents may compromise the confidentiality of the assets protected by TOE by unauthorized methods.

But this phrase is not sufficient because the threat agents and the attack methods are having the possibility to be divided.

It's very important to keep in mind that many kinds of threat agents and attack methods may exist in the 'assets protected by TOE. Therefore, the threat agents and the attack methods must be considered to contain all cases. For example, the phrase 'by unauthorized methods' is not suitable in some cases, because the 'threat agents' can contain the 'authorized users' and the authorized users may comprise the confidentiality of the assets protected by TOE by accident. So we should remove the phrases 'by unauthorized methods' and 'Threat agents', because all types of users and attack methods must be considered. We can use the threat description as like:

- The confidentiality of the assets protected by TOE may be compromised

As seen at the above phrase, in the case we use the concept of the 'assets protected by TOE', the descriptions for the threat are expressed as a kind of 'possibility' of attack. Some examples are like these:

- Malicious code may exist in the assets protected by TOE,

- Vulnerabilities may exist in the assets protected by TOE,

- Etc. 
Systems, services, and others are included in the assets protected by TOE, and systems and services may consist of resources and source codes. Therefore, they may contain the malicious codes or vulnerabilities in the resources and codes.

\subsection{Benefits}

The benefits of this method we proposed are the following things:

Simplicity: When describing the threat in the PP for security environments, many threat phrases which finally come to same security functional requirement are needed because the assets, attack methods, and the threat agents are different. Sometimes, it's possible to use the assumptions or OSPs to reduce the threats, it is not sufficient. If we use the concept 'the assets protected by TOE', we can reduce the count of the threat phrase because the reason of dividing threat may be removed.

Expandability: Because of the fast growth of technology, everyday we are faced to many new malicious codes, viruses, and security holes. If does the new asset is added in the operating environments? If does the new vulnerability is found in the source codes which consist of a service? In these cases, we can solve problems by adding a few phrases to the 'PP INTRODUCTION' part of the PP, or maybe, if we are lucky, nothing will be changed.

Comprehensiveness: In fact, the form we used does not help the reader to understand what the security need is, especially since it applies to any TOE. But this may be another benefit of the method we proposed. The method using the concept of the assets protected by TOE is applied to many security systems like as Intrusion Detection Systems (IDS), Firewall, Virtual Private Network (VPN), and so on. The assets of these systems can be divided by using same way we proposed in this paper.

It's very important to keep in mind that the method we proposed is applicable only to the assets must be protected by the TOE. The threat descriptions for the TOE should only refer to potential events which could 'directly' compromise the assets requiring protection.

\section{Conclusions}

Many kinds of PPs are developed already and they have strong theoretical rationale about the development process. But there is no consistent theory for describing the security environments especially threats. Therefore, when readers review the PPs developed by other person or company and developers write the PPs they are to use make many kinds of mistakes and feel complexity. In fact, when we develop the PPs, we can find that there are many similar things between secure systems because the IT systems which aim to protect something have the similar structures and characteristics.

In this paper we proposed a method for dividing the assets and this method will be helpful to describe the threat phrase for the PP. 


\section{References}

1. ISO. ISO/IEC 15408-1:1999 Information technology - Security techniques - Evaluation criteria for IT security - Part 1: Introduction and general model

2. ISO. ISO/IEC 15408-2:1999 Information technology - Security techniques - Evaluation criteria for IT security - Part 2: Security functional requirements

3. ISO. ISO/IEC 15408-3:1999 Information technology - Security techniques - Evaluation criteria for IT security - Part 3: Security assurance requirements

4. KISA. Information Security Systems \& Certification Guide, 2002

5. ISO. ISO/IEC 15292:2001 Information technology - Security techniques - Protection Profile registration procedures

6. ISO. ISO/IEC PDTR 15446 Guide for the Production of PPs and STs, Version 0.92

7. Russell Dean Vines. Wireless Security Essentials, 2002

8. Brian Carter and Russell Shumway. Wireless Security End-To-End, 2002

9. Randall K. Nichols and Panos C. Lekkas. Wireless Security Models, Threats, and Solutions, 2002

10. ISO. ISO/IEC WD 18045 Methodology for IT Security Evaluation

11. ISO. ISO/IEC WD 18028 Information Technology - Security techniques - IT Network Security

12. Science Applications International Corporation. Intrusion Detection System System Protection Profile, Version 1.4, February 4, 2002

13. Science Applications International Corporation. Intrusion Detection System Scanner Protection Profile, Version 1.1, December 10, 2001

14. Science Applications International Corporation. Intrusion Detection System Sensor Protection Profile, Version 1.1, December 10, 2001

15. Science Applications International Corporation. Intrusion Detection System Analyzer Protection Profile, Version 1.1, December 10, 2001

16. DGA. Protection Profile Firewall a exigences reduites, Version 2.2, 1999

17. NSA and SPARTA. U.S. Department of Defense Traffic-Filter Firewall Protection Profile For Medium Robustness Environments, Version 1.4, 2000

18. NSA. Mobile Code Desktop Draft, Version 1.0, 2000

19. NSA. Protection Profile For Multilevel Operating Systems In Environments Requiring Medium Robustness, Version 1.22, 2001

20. Schlumberger. Smartcard Embedded Software Protection Profile, Version 1.2, 1999

21. Atmel Smart Card Ics. Smartcard IC Platform Protection Profile, Version1.0, 2001 